\title{
Prevalence of Head and Neck Cancer in the State of Meghalaya: Hospital-based Study
}

\author{
Neizekhotuo Brian Shunyu, Judita Syiemlieh
}

\begin{abstract}
In India, 20 to $40 \%$ of all cancer arises in the head and neck region. The highest rate is seen in the eastern and southern regions of our country. The alarming high prevalence of head and neck cancer (HNC) in this region has prompted us to undertake this retrospective study. This study is to give the picture on the prevalence of HNC in the region, as such studies are lacking, till date. This is a 5 years retrospective study of cancer patients from 2007 to 2011 who have been registered in Civil Hospital, Shillong. The period of study is short as registry before 2007 was not properly recorded, but the high prevalence of cancer especially HNC warranted the need to undertake this retrospective study. During the 5-year period, there were a total of 3,123 cancer patients, registered in Civil Hospital, Shillong. In this study, HNC and esophageal cancer constitute 2,207 $(70.67 \%)$ cases of the total body malignancy (TBM) which is much higher than other studies done in other parts of the country. Fourth decade is the most common age group comprising of 471 cases which accounts for $32.72 \%$ of all HNC. This high prevalence of HNC in the young population of the region is alarming and hence needs a comprehensive afford to meet this challenge.

This paper, therefore, is an attempt to quantify the spectrum of HNC in the region hoping that this paper will help the health professionals to understand the burden of HNC in the region, so as to generate strategies for future planning.
\end{abstract}

Keywords: Total body malignancy, Head and neck cancer, Prevalence.

How to cite this article: Shunyu NB, Syiemlieh J. Prevalence of Head and Neck Cancer in the State of Meghalaya: Hospitalbased Study. Int J Head and Neck Surg 2013;4(1):1-5.

\section{Source of support: Nil}

Conflict of interest: None declared

\section{INTRODUCTION}

The prevalence of head and neck cancer (HNC) with respect to total body malignancy (TBM) varies from 9.8 to $40 \%{ }^{1-7}$ Its incidence is high in Asia and other less developed countries and these countries account for nearly $0.7 \%$ million new HNC every year. ${ }^{8}$ In India, HNC account for 20 to $40 \%$ total body cancer with oral cancer being the most common. The highest rate is seen in the eastern and southern part of our country. ${ }^{9}$ The morbidity and mortality associated with HNC is of grieve concern to the patients, their relatives and the population at large. Head and neck refer to a group of biologically similar cancers originating from upper aerodigestive tract. A total of 90 to $95 \%$ of HNCs are squamous cell carcinoma (SCC). ${ }^{6,10}$ HNCs are strongly associated with certain environmental and lifestyle risk factors, such as tobacco, smoking, alcohol, poor nutrition and ultraviolet light. ${ }^{4,11,13}$ There is casual relationship of 5 to 25 -fold increase in the risk in person who smokes as well as drinks alcohol. ${ }^{14}$ Thus, majority of HNCs can be prevented by societal and lifestyle changes. In Northeast India, tobacco-related HNC is very high and its prevalence is found to be higher than the other studies. In our study, HNC and the esophageal cancer account for $70.67 \%$ of TBM.

\section{MATERIALS AND RESULTS}

This is a retrospective study on the prevalence of HNC in the region carried out in Civil Hospital, Shillong, Meghalaya, of all the cancer patients registered from 2007 to 2011. During this period, a total of 3,123 TBMs were registered. HNC and esophageal cancers constitute $70.67 \%$ $(1,438+769$ cases $)$ of the TBM. HNC alone excluding esophageal cancer constitute $46.04 \%$ (1,438 cases) of the TBM and esophageal malignancy constitutes 24.62\% (769 cases).

Prevalence: Oropharynx and oral cancer are the most common HNC. Oropharyngeal cancer constitutes 24.2\% $(348 / 1,438)$ of HNC with male and female ratio of 7.92:1 while oral cancer constitutes $23.92 \%(344 / 1,438)$ of HNC with male and female ratio of 1.27:1. In hypopharynx region, the prevalence is $18.42 \%(265 / 1,438)$ of the total HNC patients with male to female ratio $(8.81: 1)$ followed by laryngeal cancer $15.61 \%(233 / 1,438)$ with male and female ratio of 7.62:1. There were 50 and 26 cases of carcinoma nasopharynx (NPC) in male and female respectively forming $5.28 \%$ of $\mathrm{HNC}$ and 25 cases of paranasal sinuses (PNS) and nose cancer cases with male and female ratio of 1.27:1. Site in relationship to the sex of the patients: Of the total 1,438 HNC cases, there were 1,118 HNC male patients and 320 HNC female patients registered in Civil Hospital, Shillong, with a male and female ratio of 3.49:1. In case of male HNC, oropharynx was the commonest site accounting for $27.63 \%$ (309 cases) followed by hypopharyngeal cancer 21.28\% (238 cases). There were 206 (18.42\%) and 193 (17.26\%) laryngeal and oral cancer cases respectively, 50 nasopharyngeal carcinoma, 14 PNS and nose cancers, 22 non-Hodgkin lymphoma (NHL), 12 Hodgkin lymphoma (HL), 10 thyroid malignancy, eight parotid malignancy, two 
submandibular malignancy and one ear cancer. Fifty-three cases were of unknown primary. Figure 1 shows the details of male HNC cases in relation to various sites. In females, oral cavity and lip were the most common site accounting for 151 (47\%) cases making it the commonest HNC in female followed by oropharynx 39 cases $(12.18 \%)$, hypopharynx 27 cases (8.43\%), larynx 27 cases (8.43\%), nasopharynx 26 cases (8.12\%), 11 nose and PNS cases, 11 thyroid malignancies, 10 unknown primary cases, eight NHL, five parotid malignancies, four HL cases and one ear cancer. Figure 2 shows the details of the female HNC cases in relation to various sites.

Age: The 4th decade was the commonest age group for the prevalence of HNC accounting for 471 cases (32.75\%) of all HNC followed by the 5th and 6th decade comprising of 346 (25.31\%) and 320 (22.25\%) cases respectively. There were 316 (21.97\%) cases in the 3rd decade while 95 patients were above 70 years and 20 patients were below 20 years. In between 20 and 40 years, there were 316 (21.97\%) cases. In the 4th decade, there were 122 oropharyngeal cancers accounting for $35.05 \%$ of the total oropharyngeal cancers, 104 oral cancers accounting for $42.62 \%$ of the total oral cancers, 97 hypopharynx cancers accounting for $36.60 \%$ of the total hypopharynx cancers and 78 laryngeal cancer cases accounting for $34.47 \%$ of larynx cancers. In the 5th decade, there were 85 (24.42\%) oropharyngeal cancers, 81 (23.54\%) oral cancers, 68 (25.66\%) hypopharyngeal cancers and 64 (27.46\%) laryngeal cancers. In the 3rd decade, there were 87 (25\%) oropharynx cancers, 65 (18.89\%) oral cancers, 54 (20.37\%) hypopharyngeal cancers and 40 (17.16\%) cases of laryngeal cancer.

\section{DISCUSSION}

The prevalence of HNC and esophageal cancer in the region is alarmingly high as it constitutes $70.67 \%$ of the TBM.

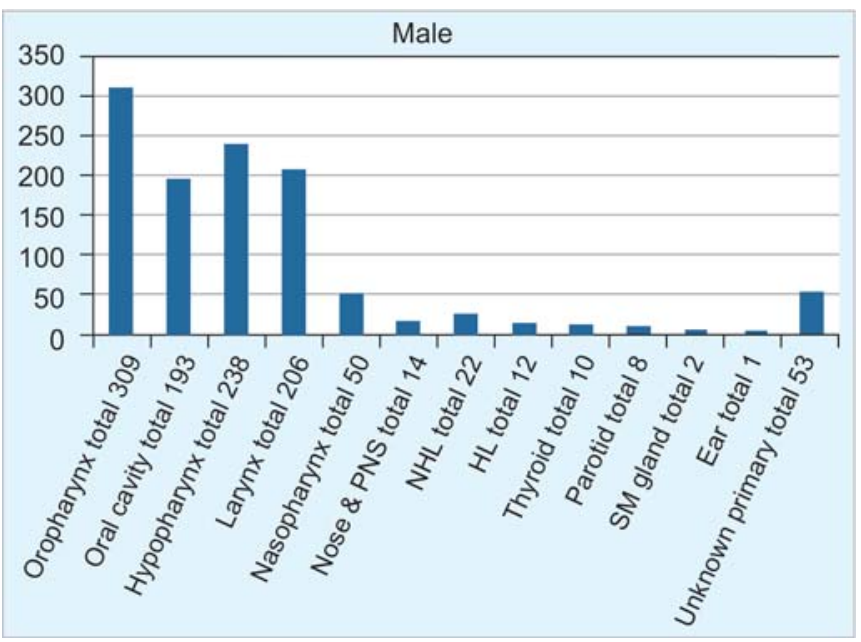

Fig. 1: Prevalence of head and neck cancer for various sites in male
HNC, excluding esophageal malignancy, accounts for $46.04 \%$ of the TBM. According to various studies, the prevalence of HNC with respect to total body malignancies varies from 10 to $40 \% .^{11,12,14,15,19,20}$ According to one of the Northeast studies, HNC and esophageal cancers account for $54.48 \%$ of the TBM. ${ }^{15}$ Such a high prevalence of HNC and esophageal cancer in the region is a major concern and is an indication of definitive important etiological factors concerning to this very region of our country. Betel nut and betel leaf chewing with lime is a common habit in this region and is socially acceptable; hence, it is chewed by almost everyone from an early age in life. The literature studies indicate that betel nut and leaf chewing is associated with an increased risk of SCC of head and neck. ${ }^{16}$ This could be one of the reasons in association with tobacco and alcohol for the high occurrence of HNC and esophageal cancer in the region.

According to literature ${ }^{6,7} \mathrm{HNC}$ is most common in the 6th and 7th decades of life but, in our study, the most common age group is 4th decade especially in cases of male patients for oropharynx, oral and hypopharyngeal cancer as tabulated in Table 1. This is something unlike the other part of the country's pattern of age distribution of HNC. The high prevalence of HNC in the young population is something to be seriously thought about. The possible explanation could be that, the early habit of betel nut and leaf chewing with lime at the young age initiate changes (prime the individual) that could possible lead to early cancer development in an individual who subsequently take tobacco and alcohol in life. Tobacco and alcohol use is high among male individuals and this could be one of the possible explanation for the high prevalent of HNC in the young population, especially among males.

In a study by Thakur et $\mathrm{al}^{7}{ }^{7}$ oropharyngeal carcinoma was reported to be the third most common (7.3\% of TBM

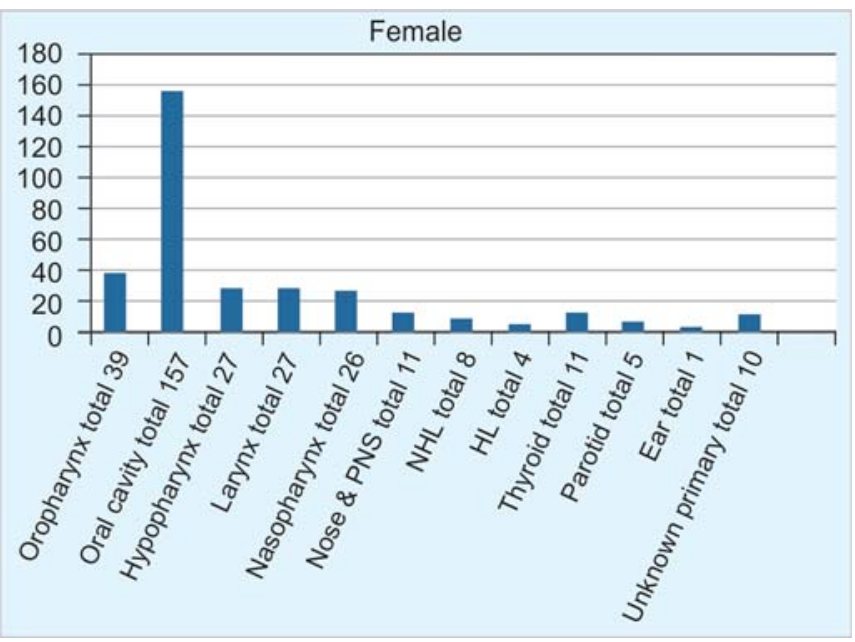

Fig. 2: Prevalence of head and neck cancer for various sites in female 


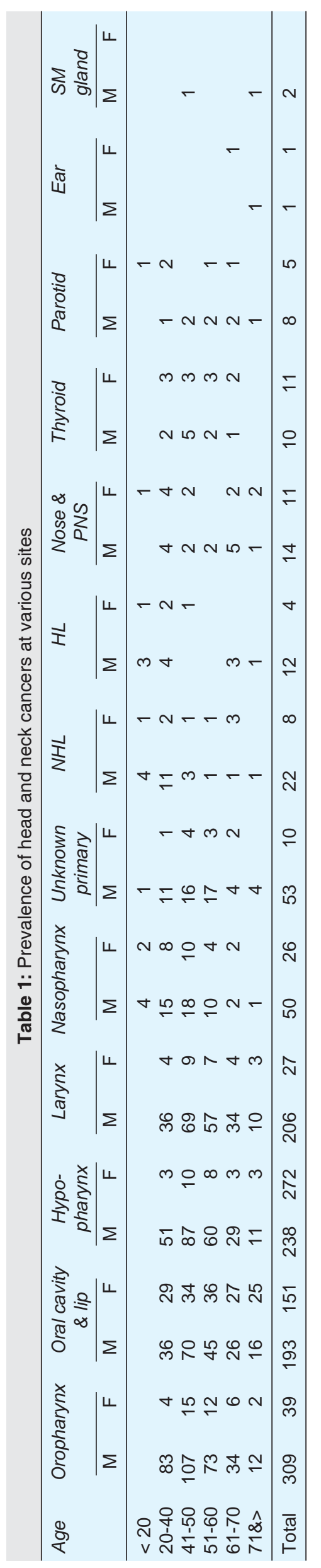


and $16.9 \%$ of $\mathrm{HNC}$ ) with male:female ratio of 3.4:1. But in our study, the prevalence of oropharyngeal cancer is very high and it forms the most common HNC accounting for $11.14 \%$ of TBM and $24.2 \%$ of HNC with male:female ratio of 7.9:1. From earlier studies, we know, human papilloma virus (HPV) is an etiological factor, especially for oropharyngeal cancer. ${ }^{17}$ There could be a possibility of high infection of HPV among the population. The infection of HPV could be high especially on the stain and abraded oropharynx mucosa as the result of the constant habit of chewing betel nut and betel leaf mixed with lime against the back ground of poor oral hygiene on an individual who also takes tobacco and alcohol. Earlier studies show oral cancer form 24 to $28 \%$ of $\mathrm{HNC}$, ${ }^{6,7,11,18}$ where tongue (49.13\%) was the commonest site involve. ${ }^{19}$ In our study, oral cancer form $23.92 \%$ of HNC with the most common site being buccal mucosa forming 37.2\% (128 cases) of oral cancer. Hypopharynx cancer comprised of $18.42 \%$ of HNC making it the third most common HNC with male:female ratio of 8.81:1. Other studies give a figure from 11.7 to $28.3 \%$ of $\mathrm{HNC}^{5-7,10}$ with a male and female ratio of 3.6:1 to 5.8:1. ${ }^{5,7}$ There is also high prevalence of hypopharyngeal cancer among the males. The reason could be the same as for oropharyngeal cancer. There were a total of 233 laryngeal cancer cases comprising $15.61 \%$ of HNC with male and female ratio of 7.6:1, while in other studies, it ranges from 11 to $26 \%$ of $\mathrm{HNC}^{4,6,7}$ with male and female ratio ranging from $3.34: 1$ to $11.5: 1 .^{4-7,18,20,21}$ Carcinoma nasopharynx was $2.4 \%$ (56 cases) of the TBM. Thakur et al ${ }^{7}$ gave a figure of $0.3 \%$ TBM whereas Bhatia et al gave NPC $8.8 \%$ of the TBM. $^{1}$ Our center get NPC cases from neighboring states like Nagaland, where NPC prevalence is high. In our study, the prevalence of PNS and nose cancer was $0.8 \%$ TBM with male and female ratio of 1.2:1. Other studies have reported carcinoma of nose and PNS in the range of 0.9 to $2.4 \%$ of $\mathrm{TBM}^{1,6,7}$ with male:female ratio between $1.5: 1$ and 2.48:1.1,5,6,22

\section{CONCLUSION}

This study aims to quantify and analyze the spectrum of the prevalence of HNC in the region. The study revealed high prevalence $(70.67 \%)$ of HNC and esophageal cancer in the young population with HNC alone excluding esophageal cancer, constituting $46.04 \%$ of the TBM. Oropharyngeal cancer was the most common HNC constituting $11.14 \%$ of TBM and 24.2\% of the HNC with male:female ratio of 7.9:1. Oropharyngeal cancer was the most common cancer in males constituting $27.63 \%$ of the HNC in male, while in females oral cancer was the most common cancer constituting $47 \%$ of HNC in female. In our study, the most common age group for the prevalence of HNC is the 4th decade, especially for oropharynx, oral and hypopharyngeal cancer in male patients. The prevalence of oropharynx, oral cavity and hypopharynx cancer was $35.67 \%$ in the 4 th decade in male HNC patients. The high prevalence of HNC in the young population needs prompt attention. We hope this study will highlight the urgent need for a comprehensive effort toward the management of HNC by the health professionals. The study also reveals the need to study the betel nut/leaf as well as HPV as the possible additional etiological factor for the high prevalence of HNC in the region.

\section{REFERENCES}

1. Bhatia PL, Jha BK. Pattern of head and neck cancers in Manipur. Indian J Cancer 1982;19:241-48.

2. Padmanabhan TK, Vasudevan DM. A statistical analysis of cancer registered at the Regional Cancer Centre, Trivandrum. Indian J Cancer 1982;19:189-96.

3. Sawai MM, Tawalkar GV, Gangadharan P. Cancer nasopharynx-a review of 1036 cases seen at Tata Memorial Hospital, Bombay. Indian J Cancer 1983;20:89.

4. Jussawalla DJ, Sathe PV, Yeole BB, Natekar MV. Cancer incidence in Aurangabad city 1978-80. Indian J Cancer 1984;21:55-62.

5. Chaturvedi VN, Raizada RM, Jain SK, Tyagi NK. Cancer of ear, nose, pharynx, larynx and esophagus in a rural hospital. J Vivekananda Inst Med Sci 1987;10:63-67.

6. Manjari M, Popli R, Paul S, Gupta VP, Kaholon SK. Prevalence of oral cavity, pharynx, larynx, nasal cavity malignancies in Amritsar, Punjab. Indian J Otolaryngol Head Neck Surg 1996;48:189-96.

7. Thakur S, Chaturvedi V, Singh AK, Puttewar MP, Raizada RM. Pattern of ear, nose, pharynx, larynx and esophagus (ENPLO) cancers in rural-based hospital. Indian J Otolaryngol Head Neck Surg 2001;53:93-99.

8. Globocan 2000: Incidence, mortality and prevalence of cancer worldwide. Lon: IARC Press 2001.

9. Parkin DM, Pisani P, Ferlay J. Estimates of the worldwide incidence of 18 major cancers in 1985. Int J Cancer 1993;54: 594-606.

10. Chakravarty S, Kar TK, Ghosh LM. Neoplasm of ear, nose, throat. Indian J Otolaryngol Head Neck Surg 1992;1:113-18.

11. Gangadharan P. Epidemiologic observation on cancer in Indian people. Indian J Cancer 1979;16:1-17.

12. Winn D. Smokeless tobacco and aerodigestive tract cancers: Recent research directions. Adv Exp Med Biol 1992;320: 39-46.

13. Iribarren C, Tekawa IS, Sidney S, Friedman G. Effect of cigar smoking on the risk of cardiovascular disease, chronic obstructive pulmonary disease, and cancer in men. N Engl J Med 1999;340(23):1773-80.

14. Murata M, Takayama K, Choi B, Pak A. A nested case-control study on alcohol drinking, tobacco smoking and cancer. Cancer Detect Prev 1996;20(6):557-65.

15. Bhattacharjee A, Chakraborty A, Purkaysth P. Prevalence of head and neck cancers in the North East: An institutional study. Indian J Otolaryngol Head Neck Surg 2006 Jan-Mar;58(1): 15-19. 
16. Jeng J, Chang M, Hahn L. Role of areca nut in betal quidassociated chemical carcinogenesis: Current awareness and future perspectives. Oral Oncol 2001;37(6):477-92.

17. D’Souza G, Kreimer A, Viscidi R, Pawlita M, Fakhry C, Koch $\mathrm{W}$, et al. Case-control study of human papilloma virus and oropharyngeal cancer. N Eng J Med 2007 May;356(19): 1944-56.

18. Kulkarni PV, Jaiswal SS, Rathod SB, Khalique A, Kulkarni RR. Profile of malignancy at Ambajogai (15 years retrospective study). Indian J Cancer 1996;33:31-36.

19. Singh NP, Sachan MS, Budhiaraj N. Anaesthetic problems in excisional surgery for malignant lesions of oral cavity and maxillary antrum. Indian J Cancer 1965;2(3):135-42.

20. Shaw HJ. Glottic cancer of the larynx 1947-1956. J Laryngol Otol 1965 Jan;79:1-14.

21. Iwamoto H. An epidemiological study of laryngeal cancer in Japan. Laryngoscope 1975;85(7):1162-72.
22. Acheson ED, Hadfield EH, Macbeth RG. Carcinoma in nasal cavity and accessory sinuses in woodworkers. Lancet 1967;289:311-12.

\section{ABOUT THE AUTHORS}

\section{Neizekhotuo Brian Shunyu (Corresponding Author)}

Assistant Professor, Department of ENT and Head and Neck Surgery North Eastern Indira Gandhi Institute of Health and Medical Science Shillong, Meghalaya, India, e-mail: drnbshunyu@ yahoo.com

\section{Judita Syiemlieh}

Senior Radiation Oncologist, Department of Radiation Oncology, Civil Hospital, Shillong, Meghalaya, India 